\title{
Precision Livestock Farming Technologies
}

\author{
Ivan Andonovic, Craig Michie \\ University of Strathclyde \\ Department of Electronic and Electrical Engineering \\ i.andonovic, c.michie@strath.ac.uk
}

\author{
Philippe Cousin, Ahmed Janati \\ Easy Global Market \\ phillipe.cousin, ahmed.janati@eglobalmark.com
}

\author{
Congduc Pham, Mamour Diop \\ University of Pau \\ congduc.pham,mamour.diop@univ-pau.fr
}

\begin{abstract}
Precision Livestock Farming (PLF) is core to satisfying the ever increasing world-wide demand for dairy products of good quality and societal concerns over animal welfare and health, whilst heavily reducing environmental load and resource use. The features of an Internet-of-Things (IoT) inspired platform with the capability to provision of a range of services that promote the adoption of precision farming principles is reported.
\end{abstract}

Index Terms - precision farming, Internet-of-Things, cloud services, livestock, location based services, LoRa, cattle rustling, dairy, geo-fencing

\section{INTRODUCTION}

The fundamental principle on which PLF is founded is that if the needs of animals and crops are satisfied at the highest granularity, then the needs of farmers and the supply chain including consumers follow. The more precisely those needs are satisfied, the less waste there is in the system, resulting in greater economic and environmental benefits. The steady decline in the number of producers and increased commercial pressures have forced consolidation within farming, resulting in the growth of the average farm size. A direct consequence is that the farmers have less time to execute traditional practices and are becoming increasingly reliant on technology. Farmers balance between sustainability and the need to invest in innovative technologies to maximise returns. Longer term, the evolution to new business models based on provisioning a range of services to the agricultural community becomes possible, fueling further the ready uptake of technology for the benefit of all operating within the supply chain.

The solutions required to support this evolution harness a number of technologies that follow the classification according to the IoT Reference Model Layers (Figure 1). Solutions rely on engineering data driven by consideration of the impacts in order for the derived product/service to be not only fit-for-purpose but can also be easily deployed and maintained with a minimum of perturbation to a sector that has followed entrenched practices for many years. The data, the heart of any solution, is 'Big' comprising many different streams of single stranded data, but markedly different from the 'Big Science Data' sets routinely encountered in drug development and oil exploration. The platform has an identifiable evolution in that the initial range of services can be extended to yield additional valuable information on a range of livestock traits of economic importance e.g. lameness, and the infrastructure in tandem with the solution development methodology can also be applied to yield products that address other market segments where data gathering and analysis monitoring improves the operational efficiencies.

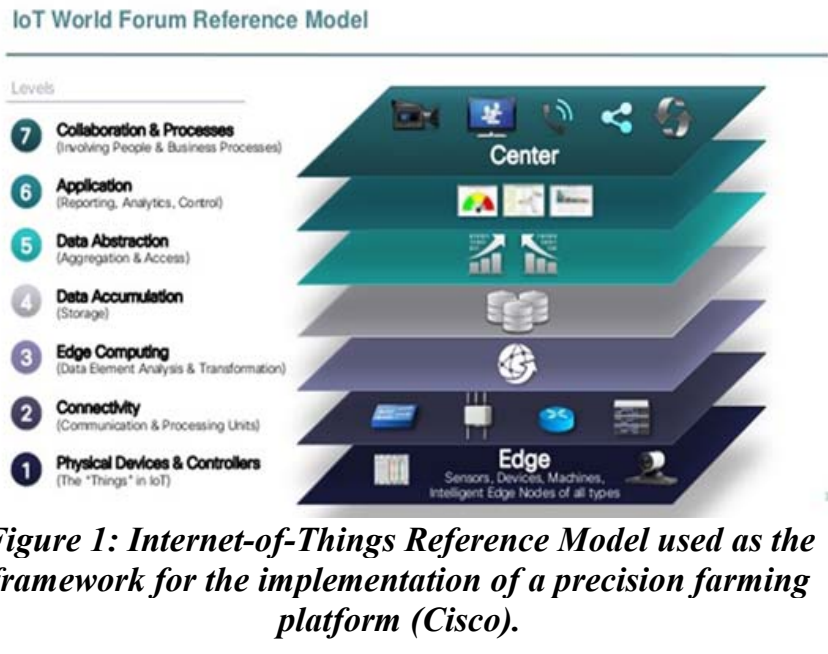

\section{BACKGROUND}

The past 20 years have witnessed a digital revolution within agriculture. The use of automated measurement methods to monitor the behaviour of animals is becoming increasingly widespread. Software techniques have been applied to recover a range of states from the measurements including rumination, eating, standing, and lying with reasonable accuracy. These states can then be the foundation for identifying key animal conditions such as the onset of illness. The approach presents significant implementation challenges as for many IoT implementation supported by a network of low power processors and wireless data downloads.

The dairy sector has increasingly adopted technological solutions to address the challenges associated with optimising operational efficiency. The steady decline in the number of producers and increased commercial pressures have forced consolidation within farming, resulting in the growth of the average farm size. In the UK for example, the number of dairy cows in the UK has fallen from 3.2 million in 1980 , to 1.8 million in 2010 .In tandem, the number of dairy producers has dropped from 35,741 in 1995 , to 15,716 in 2010 and the average herd size has risen; in 2009, the average number of cows per herd was 113 , compared to 80 in 1999. In spite of this evolution, the overall milk production 
in the UK has remained relatively constant (13319 million liters in 2008 versus 13539 million liters in 2013) reflecting the fact that farms are operating more intensively to optimise production. It is against this economic driver, that the features of an extensible IoT based platform will be detailed.

The dairy cattle-focused platform elements comprise a robust, high node count sensor network with a neck mounted collar engine gathering activity data from individual animals and a cloud based software environment that manages onfarm data and pro-actively alerts the farmer, real time of key operational and management interventions (Figure 2).

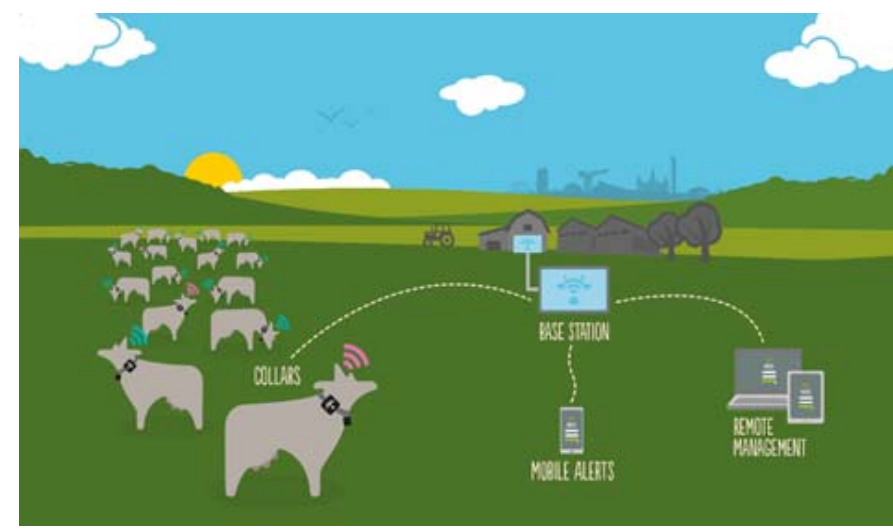

Figure 2: Schematic of the platform comprising neck mounted collar wirelessly downloading behavioural data of individual animals to a local PC and /or a cloud for presentation and decision support applications.

An electronic unit housed in a collar around the neck of an individual animals, records individual neck and muscle movements continuously using a 3-axis accelerometer. The measured data is processed on-collar using advanced software and the data is downloaded to a PC wirelessly whenever a cow enters the receiving area of a base station, located either within the field, or within the milking parlour. Alerts indicating a particular cow condition are displayed on a local PC or on the cloud. Each collar learns normal behaviour patterns and alerts the farmer of cow conditions only when there is an indication of a high degree of confidence that intervention is required, enabling the farmer to schedule a corrective action (Figure 3 ). The significant differences in the variance of measured raw accelerometer data allows the derivation of various cow states e.g. rumination and eating.

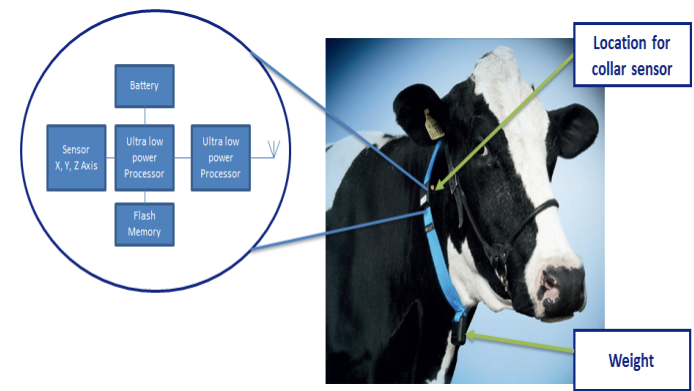

Figure 3: Smart collar unit around the neck of an animal. The unit comprises a 3-axis accelerometer, processor, low power ZigBee wireless interface and two AA batteries. The weigh ensures registration of the collar with respect to the animal's head.
The resultant mapping of the energy spent throughout the day is the basis for the unique identification of a number of individual cow conditions. required. The implementation of the advanced software directly on the collar results in a profound reduction of the volume of data required to be downloaded to the application; the solution only generates alerts, which are transmitted at critical periods. The net gain with such an approach is scalability; deployment adaptability to service various farm environments; operating lifetime is in excess of 5 years without the need for battery change.

\section{ACTIVITYAND EATING/RUMINATING}

\section{A. ACTIVITY DETECTION AND FERTILITY}

One of the strongest drivers for the ready adoption of technologies within animal monitoring has been the need to optimise the fertility of dairy herds. Throughout the world, there has been a decline in cattle fertility, the reasons for which are complex and include variables such as selective breeding practices designed to optimise milk yield and other welfare factors such as reproductive diseases (e.g. metritis and ovarian cysts). Additionally, deficiencies in management practice, poor nutrition, and poor oestrus detection significantly contribute to low pregnancy rates. The cost of cattle infertility derives from the loss of revenue from milk production. If an eligible cow is not bred (or is incorrectly bred), the farmer loses approximately 21 days of milk production estimated at around $£ 120$ per cow in the UK. The imperative to improve fertility is therefore strong and while the overall herd fertility depends on a range of factors, it is widely recognised that oestrus detection is one area where a technology may have a role to play. Historically, oestrus detection in cattle has been carried out by a skilled observer, the farmer or stockman who look for visual signs of oestrus. The success of this traditional approach is heavily influenced by the skill of the observer and the time that they have available to observe the herd. In many cases, the strong visible signs that a cow may display will occur during the night when nobody no-one is present to observe their behaviour. An examination of the efficiency of farm workers to detect heats, carried out in 1994 over 4550 herds in the US, found a mean detection accuracy of $38 \%$.

A cow standing to be mounted - the so-called, 'standing heat' -, is recognised as an accurate sign of oestrus; if a cow is not in oestrus it will generally move away quickly when mounted. This activity behaviour is a response to elevated levels of estrogen in the blood relative to progesterone which falls around ovulation. On average, a heat may last between 8 hours and 30 hours. Shorter heats are more difficult to identify by observation and may occur through the night. Secondary signs of heat are more amenable to technological solutions that can support an automated heat detection solution, for example cows coming into heat will generally be more restless, tend to eat less and spend less time lying. Such traits can be detected using activity monitors. Here a tri-axial accelerometer enables a rich set of information to be derived as inputs to detect not only walking behaviour (step counting) but can also yield the standing/lying budget which can be an indicator of other welfare events (Figure 3). 


\section{B. EATING/TRUMINATING TIMES AND WELFARE}

Monitoring the feed intake of cattle is considered an excellent good proxy for establishing a view of overall welfare; cows that are ill will spend less time eating. Furthermore, rumination, characterised by a steady rhythmic chewing action is a key part of the digestion process; a healthy cow will ruminate for 500 minutes-600 minutes per day. The chewing activity helps maintain the ruminal $\mathrm{pH}$ at a level suitable for microbial activity. Here, rumination is identified from the motion of the neck muscles, effectively deriving multiple signatures from the same collar. During an eating phase, the cow has to tear the feed (e.g. grass) from the ground, partially chew it and then swallow for remastication at a later time. Consequently, the muscle motions observed on the neck are considerably larger and this can be observed by the spread of acceleration measurements. This process allows the cows behaviour to be identified at any point in time using only a simple measurement of the acceleration on the neck only. An example accelerometer processed output characteristic of the rumination signature is derived from estimating the variance and the frequency content of the accelerometer measurement (Figure 4).
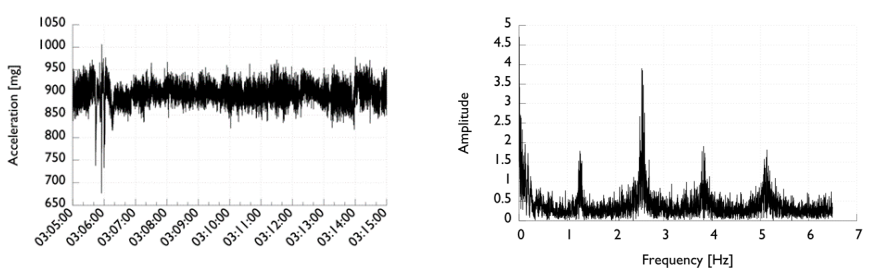

Figure 4: Time series representation (left) and corresponding frequency spectrum (right) of the processed acceleration output representing 'ruminating'.

The jaw movements exhibited during eating are however, significantly larger than those during rumination. Furthermore, eating signatures are also accompanied with a wider range of head movements. The movements of the jaw during eating are less rhythmic therefore the frequency components that are present during rumination are not observed. Figure 5 shows the higher level of variance generated during eating along with the evidence of more low frequency movements but lack identifiable frequency peaks compared to the ruminating signature (cf. Figure 4).
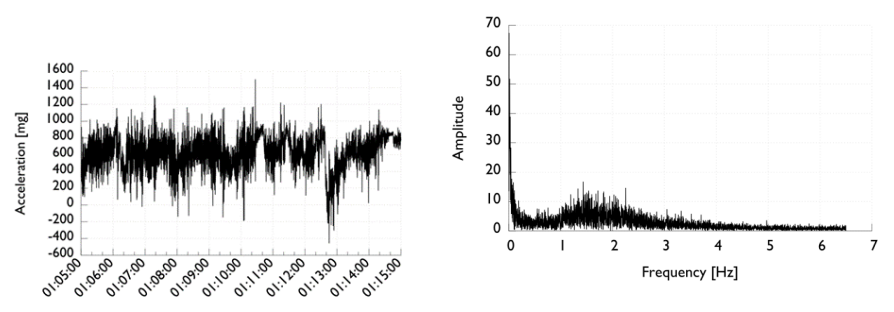

Figure 5: Time series representation (left) and corresponding frequency spectrum (right) of the processed acceleration output representing 'eating'.

Figure 6 is an illustration of a measure of the degree the cow's activity changes in a 90-minute period. As individual cows move into 'heat', their level of 'restlessness' increases and hence the variation in activity rises. The red line, is a measure of how significant this change is compared to normal behaviour, alerting the farm operative on the likelihood that a cow is ready to be inseminated. The other two traces - the 'green' and the 'dark blue' - reinforce the prediction; the 'green' trace is a measure of time the cow spends ruminating compared to the average rumination time for the past week. Since it is known that this should fall as the cow comes into heat, it provides an additional metric to reinforce the diagnosis. Figure 6 also shows that during this period the eating time - the 'dark blue' trace which maps the period of time a cow spends eating compared to the average time for the past week - increases. This is in fact a failure of the classification process to discern certain behaviour types. The cow is not eating more but will spend an increased amount of time licking and rubbing other cows with her head. These register similar muscle movements as eating and hence the graph displays an apparent increase in eating.

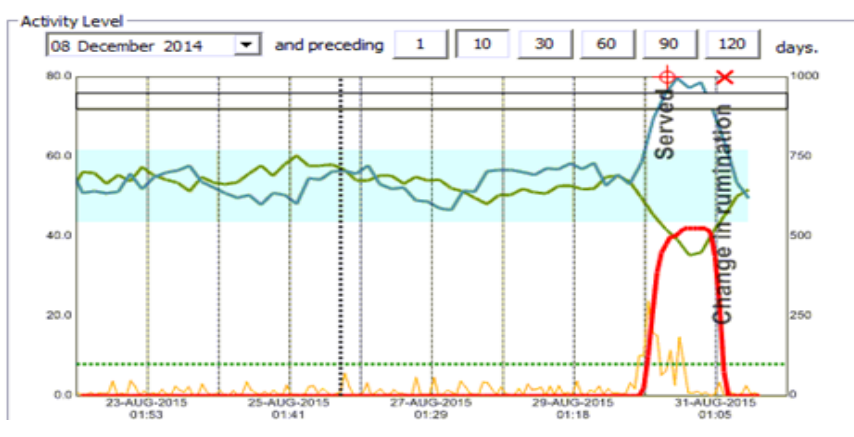

Figure 6: Illustration of a rise in activity accompanied by a fall in rumination at the point of oestrus.

\section{TECHNICAL ADVANCE ON LOCALIZATION}

Identifying the location of cattle (and other livestock) still represents a significant challenge within many farming applications. Presently there is no automated solution that provides 24-hour coverage of location with a high degree of precision (ideally within $3 \mathrm{~m}-5 \mathrm{~m}$ ) that can operate over extended periods (several years) over free grazing environments. Such capability would support the delivery of services such as;

- Fence free automated grazing management e.g. strip, cell or rotational grazing

- Automated cattle mustering

- Provision of animal theft deterrent

- Alerting farmers to fence breaks

- Identifying the location of calving (or ill) cattle

- Guiding cattle away from environmentally sensitive (or dangerous) areas.

There are many GPS solutions for cattle management but to date, GPS-based systems have been overly power intensive to support long term deployment. Recent developments in wirelessly enabled positioning systems have lacked the scale of penetration (base station infrastructure) but show great promise. The usage of recent LPWAN (LowPower Wide Area Networks) technologies such as Sigfox [1] and LoRa [2] offer potential solutions of value. As Sigfox positions itself as an operator, Sigfox-based devices need annual subscription and their usage is tightly bounded to the 
presence of the operator. LoRa is a more open technology, requiring only the radio parts to deploy ad-hoc LoRa networks.

A low-cost GPS-based collar for cattle management has been developed for deployment in sub-Saharan Africa countries [4]. The collar tracks the position of cows but can also be used to prevent cattle rustling, an important service in many developing countries (Figure 4). The collar design draws on the generic IoT platform technologies where a lowcost micro-controller such as the Arduino Pro-Mini supports the software consisting of various building blocks for realworld IoT deployment use cases e.g. data encryption, physical sensor management. An efficient integrated antenna [3] avoids any external fixings.

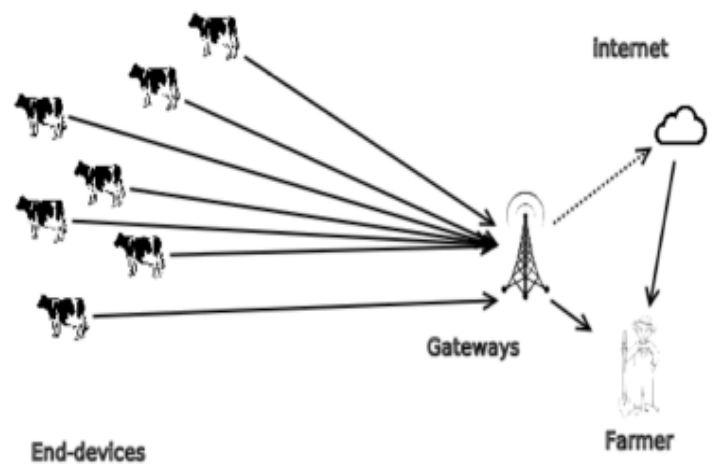

Figure 4: Illustration of the architecture used for geolocalization.

The default behavior of the GPS collar is as follows; (1) wake-up every 30 minutes and secure a GPS fix during a maximum time of $30 \mathrm{~s}$, (2) a beacon is transmitted to the gateway including the GPS coordinate if a GPS fix is available. At the gateway, a list of active collars can be maintained and if their coordinates are available a web interface will display their relative position to the gateway. The GPS module draws about 50mA when trying to get a fix. To minimise energy consumption, the module is deep sleep. Between 2 fixes, the whole system is in deep sleep mode, consuming only $5 \mathrm{uA}$. The first GPS fix typically needs $25 \mathrm{~s}-$ $28 \mathrm{~s}$ while subsequent fixes usually need $4 \mathrm{~s}-7 \mathrm{~s}$. The transmission of the message consumes about $40 \mathrm{~mA}$ during $2 \mathrm{~s}$ Therefore, with a periodic message every 30 minutes and a mean fix time of $5.5 \mathrm{~s}$, the mean energy consumption per hour is around $0.202 \mathrm{~mA}$. The collar is powered by $2 \mathrm{AA}$ batteries $(2500 \mathrm{~mA})$ and thus the lifetime is around a year.

\section{FUTURE APPLICATIONS}

Cattle rustling is still remains one of the biggest problems that faces farmers throughout the world resulting in significant financial losses. Other major challenges are losses owing to attacks from predator animals such as wolves and in certain areas of the world, the increasing number of the animals shot during hunting seasons.

\section{A. CATTLE RUSTLING}

A number of solutions that address the prevention of rustling have emerged but none provision (1) information on cow conditions (2) location in real time and (3) long range connections at low power consumption. The combination of these features will have a positive impact in terms of increasing dairy production and the security of the food supply. The integration of an accelerometer with location technologies in one unit offers an enhanced rustling deterrent. Any perturbation sensed by the accelerometer which is a departure from 'normal' behaviour, will issue an automatic alert and together with an accurate location, can empower rapid response and prevention. The additional challenge faced in these environments is long distance connectivity. A robust, reliable and long distance link between the collar (mounted on a free grazing animal) and a gateway to the Internet is a necessity. In many cases, the bandwidth required may not be significant but operational range of 10's of kilometers is a necessity. One option that has the characteristics to meet such operating conditions is LoRa technologies, the most recent of which (LoRaII) provides the potential for deriving location through triangulation of packet arrival time via highly synchronized base stations. The precision of location delivered by LoRaII (10m to $30 \mathrm{~m})$ may be adequate for some applications (e.g. locating ill or calving cows) but will not in itself, provide sufficient accuracy to support fence-free automated grazing for example.

The LoRa protocol was specially designed for IoT applications and offers improved features in comparison with its rival LPWAN technologies; significant battery life, network range, capacity and low cost. The set of features is highly desirable in agricultural settings enabling a network that can support thousands of nodes, one for each cow. The data collected from collars is forwarded through the Gateway(s) to a network server or onto a cloud platform for onward dissemination through a number of established channels e.g. smartphone (Figure 5).

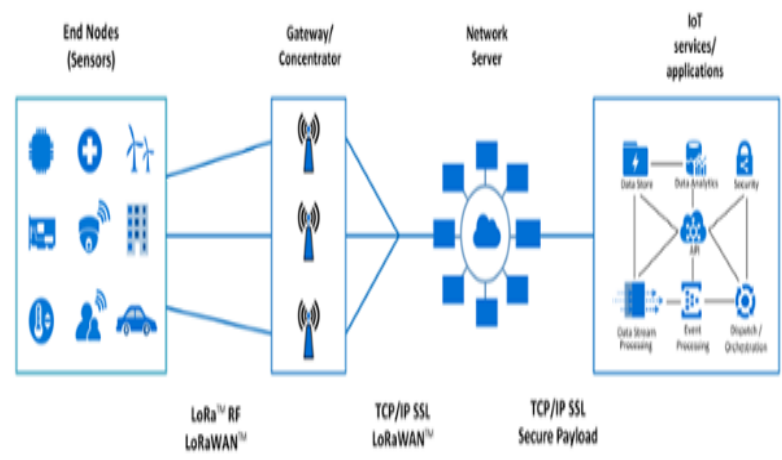

Figure 5. Illustration of the architecture of the LoRa network.

The low power consumption with a coverage range up to $15 \mathrm{~km}$ in rural environments of the resultant network are key; the use of energy harvesting technologies such as a solar panel integrated onto to the collar will increase further the autonomy of the solution.

\section{B. LOSSES DUE TO PREDATOR ATTACKS}

Loss of sheep is a significant issue which has impacted many farming concerns with concomitant financial losses. In France in 2015 only, the number of sheep attacked by wolves is estimated at 10,000 [4]; the problem is projected to become even more acute over the next few years as the 
number of wolves is estimated to increase. The self-same solution as described for rustling prevention, can serve this application. The behaviour of animals will change dramatically and immediately, once an animal senses danger such as a wolf approaching. In terms of a flock, several sheep will react simultaneously, another indicator of a critical scenario. It may be argued that similar behaviours might be caused by other natural phenomena such as fire but their temporal profiles are different. The solution may also offer different types of alerts defined to distinguish between level of dangers; each class of alert triggers the most appropriate standard operating response.

\section{LOSSES DUE TO HUNTING}

Many farmers suffer significant financial losses as a consequence of hunting which can also lead to aggressive disputes between parties. This application is yet another possible use for the solution, acting as a source of reliable information to aid arbitration. The potential to implement virtual fencing using location information also exists ensuring that the animals in question are restricted to a specific location, in so doing demarking areas. An alert can be issued immediately to the farmer in the event an animal strays outside of the designated filed. Indeed, other means e.g. integrating an electric shock mechanism within the collar would physically control the area where the animal is free to graze.

\section{PLATFORM EVOLUTION}

Integration of multiple sensor strands offers the potential for greater precision in detection of critical animal events and opens up the potential for delivering additional benefits and services through collaborative platforms exchanging and sharing data. For example, feeding wagons can improve overall herd nutrition by delivering a uniform mix of feed that does not vary in composition along the length of the feed bunk or from day to day. Hence all animals are fed equally. In addition, are beginning to integrate spectroscopic measurement instruments onto feed wagons to analyse feed composition at the point of delivery. This information, along with time and quantity of feed dispensed, is relayed to a cloud server. This opens up the possibility for nutritional experts to analyse the relationships between feed composition and production outputs on a real time basis. Ultimately, individualised feed optimisation strategies can be developed provided that accurate estimates of individual feed intake can be derived. A challenge remains to be addressed in this context that while feeding times may be accurately determined and related to feed intake on a per animal basis, feeding rates are animal specific and require a method to individual calibration. This is a challenge for a production environment. For the near term therefore it is likely that the fusion of multiple data streams will have the greatest impact in improving the diagnosis of welfare conditions related to changes in more than one variable.

\section{CONCLUSIONS}

Distributed intelligent sensing networks - IoT networks - from physical front-end devices, the underpinning technologies such as signal processing, networking, information dissemination, through to advanced diagnostics are at the heart of the deployment of a range of services which positively impact many international industrial sectors. The platform reported in the paper focusses on the extremely important sector of farming and embodies all of the elements of the traditional IoT technology stack (numbers reference the IoT Reference Model displayed in Figure 1);

(a) e.g. accelerometer based neck mounted senor is the measurement engine (1)

(b) low power wireless connectivity (2)

(c) e.g. on collar processing based on artificial intelligence software that reduces the volume of data that requires to be transmitted (3)

(d) data accumulation either at a local on-farm PC or the cloud (5)

(e) visualisation of the key conditions of individual animals (5)

(f) e.g. fertility and health service (6)

(g) multiple log-in capability so that other members of the supply chain can remote access to the information e.g. vets, AI (7)

A number of sector-specific engineering challenges dominated the design of the platform; ease of installation and maintenance, scalability in terms of the number of animals managed, location and geography independence, adaptability in service provisioning, multiple interfacing/dissemniation channels and power consumption. All of the above requirements were captured through detailed knowledge elicitation exercises with an extended agricultural community at the outset and drove the implementation of the solution.

The sole source of data is a neck mounted low cost accelerometer; the raw measurements are pre-processed on the collar in order to minimise transmission bandwidth and preserve power. The processed collar data is forwarded to a central data accumulation location where additional processing is carried out to identify key features from the acceleration signals and in turn generate animal conditions. Based on user input, three key conditions were highlighted as being of near term value viz. the onset of heat, the time spent eating and the time spent ruminating. The characteristic features in terms of variance and frequency spectrum formed the basis of the accurate identification of these three states. The information is then presented to the user(s) in a manner that is easy to absorb and inform an immediate action.

The platform enables the management of farming environments of significant scale (1000s of sensor nodes) to operate and optimises the power consumption of each node such that the battery lifetime exceeds 5 years. The information presented locally, on-farm to the farmer is subsequently synchronised with a cloud-based environment that allows the farmer to interact with the farm regardless of his geographical locations and empowers the supply chain to increase the overall production efficiency of the sector.

The integration of multiple sensors into what is effectively an operational decision support platform results in a greater benefit than that derived from the individual sensors alone. For example, each additional sensing modality will enhance the understanding of the practices across the farming environment and render any decision support service more robust in its diagnostic capacity. Multiple sensor outputs can be mined for variances (both individual and combined) that positively indicate the efficiency of any process. 


\section{REFERENCES}

[1] Sigfox, https://www.sigfox.com/en

[2] Semtech LoRa. LoRa modulation basics. rev.2-05/2015 and https://www.lora-alliance.org/

[3] Waziup, http://waziup.eu

[4] FairFax Agricultural Media, Mathew Cawood, http://www.farmweekly.com.au/news/agriculture/general/new s/wolves-and-sheep-dont-mix/2751939.aspx 